\title{
REMARKS CONCERNING REFERENCE FRAMES
}

\author{
James A. Hughes \\ U.S. Naval Observatory \\ 34 th and Massachusetts ave., NW \\ Washington, D.C. 20392
}

Twenty years ago I published A Catalog of 939 PZT Stars on the System of the FK4. At the time it was well received and indeed it did tighten up various PZT results. As I recall, the catalog, among other things, confirmed an empirical relationship between the Richmond, Florida and Washington, DC instruments which had been derived by Prof. Markowitz. In any event, I planned to follow up on this work, but an assignment to E1 Leoncito, Argentina intervened. Perhaps it was just as well because other things were happening. Astronauts were soon tramping about the moon and littering up the place with a used car and something called a retro-reflector. Radio astronomers were constantly refining the astrometric potential of their interferometric techniques; strange, high-z, starlike objects had been detected a few years earlier; shiny, new satellites were launched; and shortly the science of determining Earth Rotation Parameters (ERP), or "Orientation Parameters" if you prefer, was to explode with vitality and to reach precisions and accuracies never before achieved. As a matter of fact, the new riches became so numerous that, like the proverbial kid in the candy store, the ERP community was, to some extent, forced to choose among some of them. Thus was born the MERIT campaign.

Regardless of the technique used, it is obviously necessary to work within a well-defined framework involving models and a reference frame, if consistent and comparable results are to be obtained. Thus, the choice of a generally accepted, highly accurate and available celestial reference system is crucial. That is why we are about to hear an impressive collection of papers dealing with the subject of celestial reference frames. The subject is central, and certainly worth devoting significant time to its discussion.

There are essentially two generic, operational choices possible when defining a celestial reference system: i.e., a dynamically based or an extra-galacticaly based system. (I note that the word "dynamical" means different things to different people and here I refer primarily to star catalogs based upon absolute observations.) The two choices are often 
referred to as "optical" and "radio" systems respectively, but I prefer to use these latter words as modifiers of the prime words, "dynamical" and "extra-galactic." After all, a radio dynamical system is certainly conceivable, witness the VLA observations of minor planets, and vice versa. Similarly, pulsar timings contribute to a dynamical system. Parenthetically may I quickly add that these are merely personal observations, and that I do not advocate investing very much learned committee time in concocting names. Such efforts in the time domain, for example, have on occasion produced mixed results.

Neither a dynamical nor extra-galactic system is some sort of static construct. They both evolve. They must, and moreover they do so independently of, and in addition to, any changes brought about by, for example, increased precision. The transition from general theories to numerical integrations for the solar system, in the case of a dynamical reference, is a typical case of such evolution. Quantities which were formerly explicitly defined, e.g., by Newcomb's theory, now become a bit eel-1ike. Slippery. How does one characterize the mean orientation of the earth's orbital plane? Various procedures, all tenable representations of the dyamical situation, can give very different results. The evolution of an extra-galactic reference is today perhaps more straightforward. Now, one is concerned primarily with the choice of objects to be included. Beyond that, questions involving the time and wavelength dependence of the direction to "the source" come into play and provide feedback to the source selection process. As such reference systems grow and more experience with them accumulates, it is not unlikely that the evolutionary process will become more complex. As a matter of fact, this complexity will probably result in a recommitment to the old, but never dead, symbiotic relationship between astrophysics and astrometry. From the point of view of positional astrometry, for example, a radio star could be a pathological object, but on the other hand, the astrometric behavior of such objects can shed light on the physics involved which immediately helps the astrophysicist to theorize and to make predictions and models. In short, to provide feed-back and forth.

In any event, for the various purposes of astronomy in general, both of these kinds of reference systems will be with us for any foreseeable future. Thus the relationship, or relationships, which exist between systems become very important. Such a relationship is usually stated in terms of some kind of transformation procedure and such transformations can range from the application of a few global constants to the use of a complete, object by object, or place by place transformation matrix. The development of these transformations requires painstaking observations of stars, planets, radio stars, pulsars, galactic cores and quasars, using practically every tool in the astrometric kit. Such transformations are usually a function of time and for that reason it is crucial that all observers report the epoch of their observations. This applies to both optical and radio observations.

The precision of VLBI observations of ERP and the demonstrated accuracy 
which has followed, place this technique one or more orders of magnitude beyond that reached by current optical techniques, and as far as a general celestial reference system is concerned, it would appear that ERP and VLBI is truly a marriage made in the heavens. Of course all this accuracy does not come free. One must now concern oneself with heretofore neglected effects such as so-called "second order" classical effects, or the subtle properties of relativistic spacetime. Of this we shall no doubt hear more.

An important consideration is that the ERP community, which was formerly a consumer of celestial reference frames, has become a producer of such frames. As a matter of fact, the continuous observations required for ERP make the process preeminent as far as establishing a tight, extra-galactic reference frame of carefully selected and researched sources is concerned. The extension of such an extra galactic frame to brighter optical objects is currently a matter of much concern and discussion in the astrometric community, and if myriad, ad hoc transformations are to be avoided, an ERP VLBI frame is crucial. Even with such a frame there remain formidible operational problems to be overcome if a more widespread, operationally useful, extra-galactic reference frame is to be realized.

Finally, I would point out that technology never stands still, and even now the first steps have been taken in optical and IR interferometry and careful proposals are being made for space astrometry projects with stated goals of sub-milliarcsecond and, dare one say it? even microarcsecond precisions. Thus it may well happen that in the year 2006 one of you younger people here will stand and say, "...twenty years ago I published a VLBI catalog of 59 radio sources. At the time it was well received, but..." I leave it to you and to your intelligence and industry to finish that future sentence. 